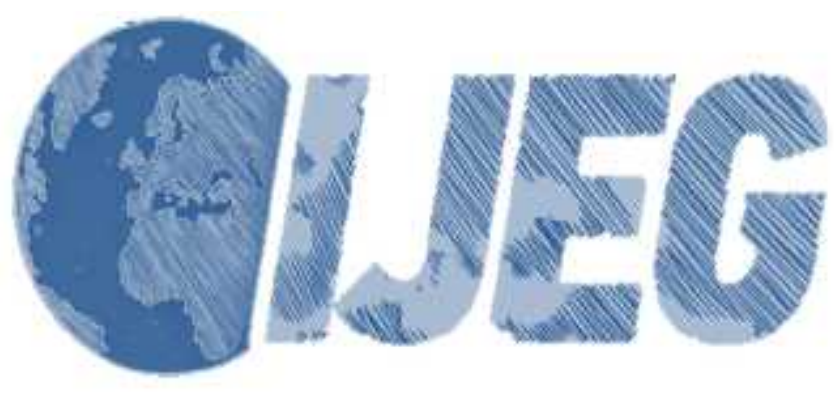

International Journal of Engineering and Geosciences (IJEG),

Vol; 3; , Issue; 1, pp. 001-005, February, 2018, ISSN 2548-0960, Turkey, DOI: 10.26833 /ijeg. 333686

\title{
DETECTION OF FOOT BONE ANOMALY USING MEDICAL PHOTOGRAMMETRY
}

\author{
Catal Reis, H. * \\ Department of Geomatics, Faculty of Engineering and Natural Sciences, Gumushane University, Gumushane, 29000, \\ Turkey \\ (hcatal@gumushane.edu.tr, hatice.catal@yahoo.com.tr)
}

ORCID 0000-0003-2696-2446

*Corresponding Author, Received: 09/08/2017, Accepted: 06/09/2017

\begin{abstract}
Photogrammetry has been used for medical diagnostic and treatment. Mostly used medical photogrammetric techniques are Ultrasound, Computed Tomography (CT) and Magnetic Resonance Imaging (MRI) images. CT and MRI are the most effective method for the early detection of foot and ankle anomaly. Researchers have been developing various methods to detect anomaly. Many image segmentation techniques are available in the literature. Computer Aided Diagnosing (CAD) system has been proposed in this study for detection of foot bone anomaly by the analysis of CT images. In this study, a segmentation based on edge detection method is proposed for the classification of anomaly in foot CT images. Edge detection algorithms are the most commonly used techniques in image processing for edge detection. Canny edge detector is evaluated in this study.

In this study, ".dicom" medical image standard format and ten male patient's foot CT images (245 images and 50 test data) are used. The used parameters are detector collimation of $64 \mathrm{~mm}$, scanning thickness of 1-5 mm, and pixel sizes of $512 \times 512$ in radiometric resolution of 16 bits' gray levels.
\end{abstract}

The proposed method consists of five major steps: (i) calculating the horizontal \& vertical gradient, (ii) determining gradient magnitude and gradient direction, (iii) applying non-maximal suppression, (iv) computing high and low thresholds, (v) hysteresis thresholding are applied to the multi-detector computed tomography to detect the bone anomaly.

In this study, automatic edge-based digital image processing techniques are applied to detect of foot bone anomaly. The proposed canny segmentation method enables users segment anomaly in MDCT of foot very quickly and efficiently. The results demonstrate that the proposed segmentation method is effective for segmenting anomaly. The proposed method obtains satisfactory performances in terms of accuracy and F-measure the area under Receiver Operating Characteristic curve (ROC curve (AUC)). The proposed segmentation method achieves an accuracy of 0.86 and Fmeasure of 0.92 , respectively.

The purpose of our study is to detect the anomaly of the foot and it was the simplest and less time consuming process.

Keywords: Medical Photogrammetry, Medical Image Processing, Segmentation, Anomaly, CT 


\section{INTRODUCTION}

The medical photogrammetry, being non-invasive methods are, apart from their geomatics applications, very convenient and precise tools for measuring medical forms and functions, as well as shapes, locations and three-dimensional of anatomic organs, area, volume and their changes in time. Medical photogrammetry generally uses medical images. Medical imaging is varied: computed tomography (CT), magnetic resonance imaging (MRI), ultrasound, angiography, and others. CT that used in this study, becomes a key tool for any theoretical framework and applied examination. In medical imaging, many applications require the classification of images in different anatomical regions: bone, muscles, organs and detection of pathological areas, such as tumors or anomaly lesions.

Anomaly lesions of the foot and ankle are rare (Foo and Raby, 2005; Bakotic and Huvos, 2001; Mascard et al., 2017). Several diagnosis methods have been proposed for anomaly/tumor detection. MR and CT imaging methods are commonly used for diagnosis (Hasbek et al., 2013). However, these imaging and diagnosis systems are not sufficient alone to meet accurate and reliable results. Second opinion is essential to avoid inadequacies and additional requirements of imaging systems and technicians and physicians failures. Therefore Computer Aided Diagnosing (CAD) is proposed for diagnosis and treatment (Lodwick et al., 1963; Toriwaki et al., 1973). CAD is applied widely in the detection and diagnosis of many types of abnormalities in medical images obtained in examinations by use of different imaging techniques (Giger et al., 2000). CAD is a term that established by taking into account equally the roles of physicians and software, whereas automated computer diagnosis is a concept based on computer algorithms only (Doi, 2007).

Edge detection can be used in many fields such as in medical field. Canny has proposed a new method of edge detecting (Canny, 1983; Canny 1986), which is the best one to step-type edge which is influenced by noise. Canny edge detection algorithms are the most commonly used techniques in image processing for edge detection. Canny edge detector is evaluated in this study.

In this study, most convenient segmentation method has been searched to detect anomaly of the foot in CT images. Matlab platform has been used for anomaly segmentation.

The manuscript of the paper is organized as follows: 1. Introduction, 2. Segmentation, 3. Bone anomaly, 4. Methods, 5. Conclusions. These five steps are described in more details as the following sections.

\section{SEGMENTATION}

The image preprocessing is an important step before doing further the segmentation of anomaly. Image noise is removed before segmentation. Segmentation is the most important part in image processing (Yogamangalam and Karthikeyan, 2013). The accurate segmentation of foot anomaly is an essential step for subsequent feature detection step. Image segmentation is the process clustering or dividing an image into multiple homogeneous regions and simplifying and obtaining searched object from original image (Kizilkaya, 2008).

Image segmentation algorithms generally are based on one of two properties of intensity values: similarity and discontinuity. Edge detection is the most common approach for detecting meaningful discontinuities in gray scale by far (Gonzalez and Woods, 2007).

Edge detection defines object boundaries within images and basically it is an image segmentation technique. The edges and corners in images can be found by detecting changes of image brightness or discontinuities sharply (Kizilkaya, 2008).

Segmentation can be classified as follows:

- Region Based

- Edge Based

- Pixel Based

- Feature Based Clustering

- Model Based

Edge detection techniques are done through detecting the maximum value of the first derivative or zero crossing of the second derivative. The representative first order differential operators (Roberts, Prewitt, Sobel, etc.) and second order differential operators (Laplace, LOG, etc.) (Rong et al., 2014). In (Biswas and Sil, 2012; Kabade and Sangam, 2016), the three main principles of the canny edge detection are as follows: low error rate, good localization of edge points and one response to a single edge.

Evaluation of the images showed that under noisy conditions, Canny, LoG, Sobel, Prewitt, Roberts's exhibit better performance, respectively (Maini and Aggarwa, 2009). In (Yuksel and Borlu, 2009), the Canny's edge detector was used to find the best region growing method for medical image segmentation (Biswas and Sil, 2012). Canny edge detection algorithm is used in this study.

The flowchart of the proposed method is shown in Figure 1. 


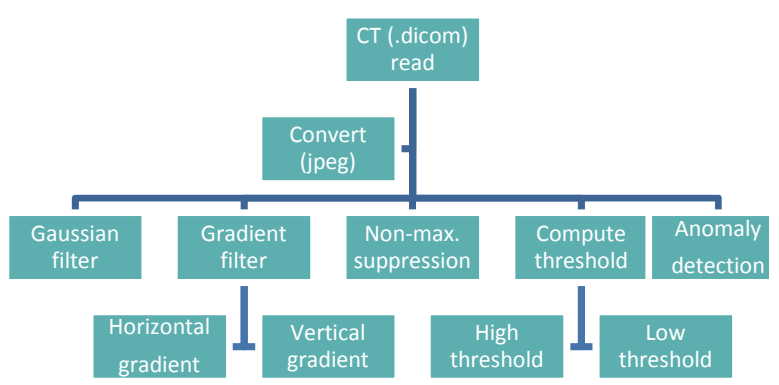

Figure.1 Canny edge detection flowchart

Algo ithm:

1. Coavolve with gradient lilters (at meltiple scales);

$$
\vec{i}(i) \equiv\left(\mathbf{R}_{\mathbf{x}}(i t), \mathbf{R}_{\mathbf{y}}(i t)\right)=\nabla \mathbf{G}\left(i t ; \sigma^{2}\right) \cdot \mathbf{I}(i t)
$$

2. Compute response magnitude;

$$
\mathbf{S}(i)=\sqrt{\left(\mathbf{R}^{2} \mathbf{x}(i)+\mathbf{R}^{2}(i)\right)}
$$

3 Compute local-edge crientation (reoresented by unit rimal):

$$
\begin{aligned}
& \overrightarrow{i n}(\vec{X}) \\
& =\left\{\begin{aligned}
\left(\mathbf{R}_{\mathbf{X}}(i), \mathbf{R}_{\mathbf{y}}(i)\right) / \mathbf{S}(i \bar{i}), & \text { if } \mathbf{S}(\overline{\mathrm{X}})>\text { threshold } \\
\mathbf{0}, & \text { otherwise }
\end{aligned}\right.
\end{aligned}
$$

4. Peak detection (non-maximum suppression along edge normal).

5. Non-maximum suppression through scale, and hysteresis thresholding along edges (Jepson and Fleet, 2009). You can see (Canny, 1986) for algorithm details.

In the presented study, canny edge detector has been used to obtain anomaly segments and their boundaries.

The bone anomaly can be divided into two groups, namely primary and secondary (metastatic) tumors (Rice et al., 2014) and they are rarely encountered (Foo and Raby, 2005; Bakotic and Huvos, 2001). The general classification of bone tumors is given by (Ozer et al., 2014) as below :

- Benign bone tumors

- Benign/aggressive bone tumors

- Malignant bone tumor.

\section{METHODS}

Computed Tomography images are obtained from Faculty of Medicine, Bezmialem Vakif University. Digital medical image format is DICOM (Digital Imaging and Communications in Medicine). CT images of ten male patient's foot ( 245 slices and 50 test data) are used. CT image stack is given in Figure 2.

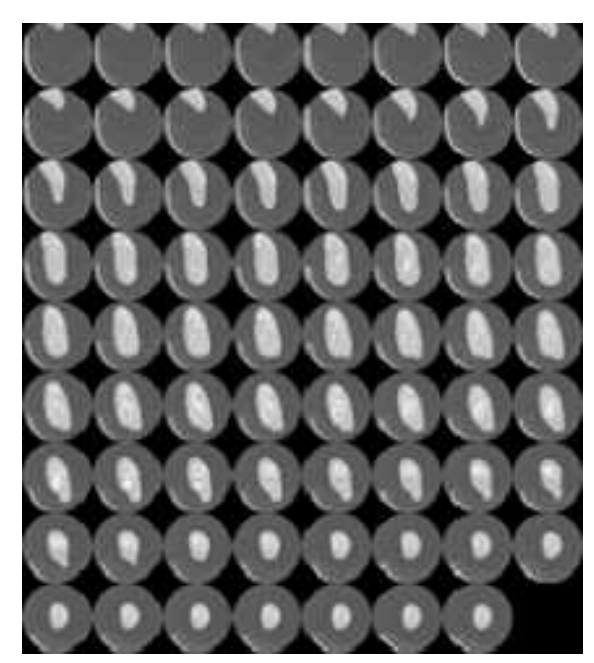

Figure.2 CT Images stack

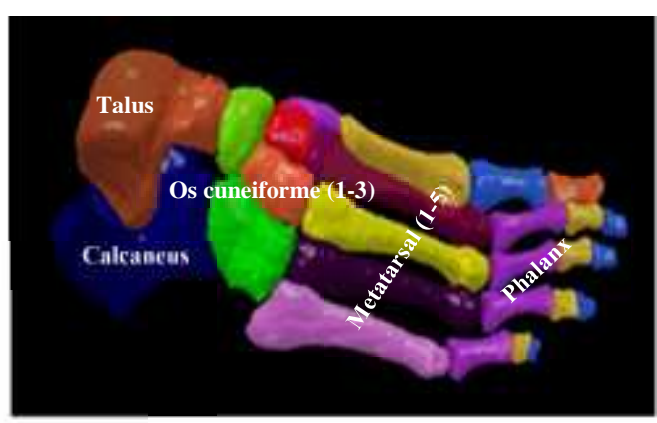

Figure.3 3D Foot anatomy

The used parameters are detector collimation of $64 \times 2 \mathrm{~mm}$, scanning thickness of $1-5 \mathrm{~mm}$, pixel sizes of $512 \times 512$ in radiometric resolution of 16 bits' gray levels. The axial images in Digital Imaging and Communications in Medicine format were first transferred into a personal computer.

Proposed Algorithm;

Step 1: CT (.dicom) image read

Step 2: Calculating the horizontal \& vertical gradient

Step 3: Determining gradient magnitude and gradient direction

Step 4: Applying non-maximal suppression

Step 5: Computing high and low thresholds

Step 6: Hysteresis thresholding

Step 7: Output

Figure 4 shows (a) the CT .jpeg image and (b) 3D of foot in the window.

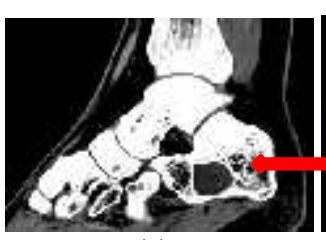

(a)

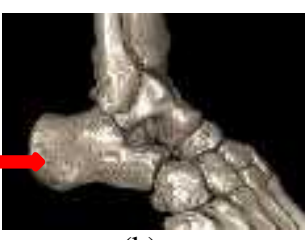

(b)
Figure. 4 CT .jpeg image and 3D model

Figure 5 shows the CT raw data in the window, the edge detection results of the canny in the slice has been 
given in Figure 6.

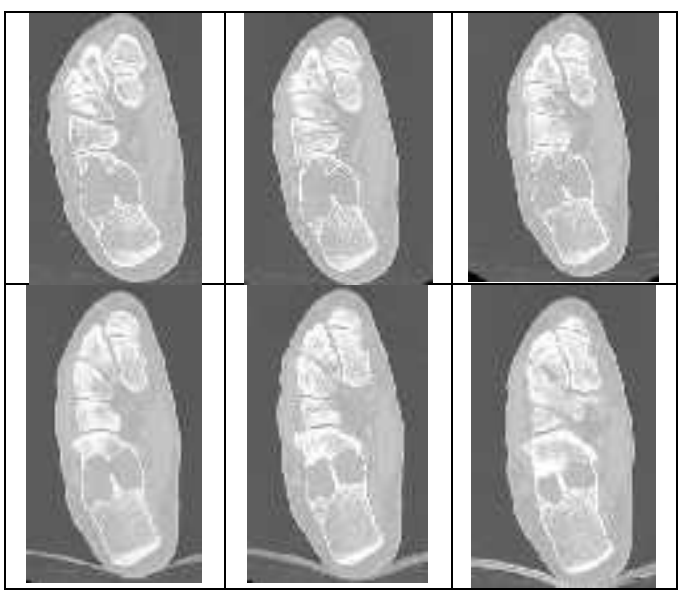

Figure.5 Original CT raw data

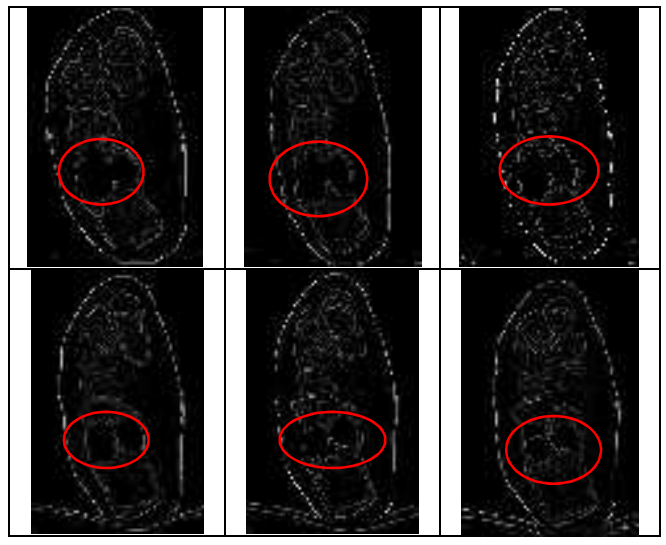

Figure.6 Examples of segmentation results by the proposed segmentation algorithm (Close-ups of the improved canny segmentation results (red))

A flowchart of the proposed method is shown in Fig. 6 and Fig.7. Original CT image and anomaly-background. The red frame indicates the anomaly, respectively. If there are two or more closed areas, there is anomaly in that bone.

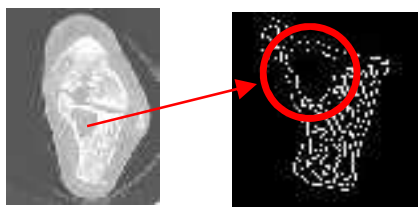

Figure.7 The flowchart of the proposed method

Matlab is used for image processing and detecting anomaly. In this study, detecting the anomaly and segmentation of the tumor and tumor-like lesion have been performed. Canny edge detection algorithm has good performance for edge detection, localization and only one response to a single edge.

In this study used receiver operating characteristic curve (ROC) for accuracy analysis. The measure of accuracy is the area under the ROC curve, often used as "AUC" for area under curve. The AUC is global a summary measure (Obuchowski, 2005). The method obtains satisfactory performances in terms of accuracy and F-measure the ROC.

$$
\begin{aligned}
& \text { Accuracy }=\frac{\mathrm{TP}+\mathrm{TN}}{\mathrm{TP}+\mathrm{TN}+\mathrm{FP}+\mathrm{FN}} \\
& F-\text { Measure }=\frac{2 \mathrm{TP}}{2 \mathrm{TP}+\mathrm{TN}+\mathrm{FN}}
\end{aligned}
$$

where; TP is the number of true positives, TN is the number of true negatives, FP is the number of false positives and FN is the number of false negatives.

To verify the proposed classification method, a comparison experiment with different two of test samples is performed. 50 different of image samples are selected for testing in this experiment and divided into two: anomaly and normal. After these process, 245 images were processed in the algorithm in order. The images are divided into anomaly or not. After this, roc analysis was done.

\section{RESULTS AND CONCLUSION}

This study proposes edge-based segmentation method for automatic bone of foot anomaly segmentation on CT images. An accuracy of 0.86 and F-measure of 0.92 are obtained, respectively. Since edge detection is an important step in digital image processing, it is necessary to point out the true edges to get the best results from the image process. So it is important to choose edge detector that suitable best to the application. Canny edge detector method enables users segment anomaly in MDCT of foot too quickly and efficiently.

Canny edge detection algorithm is one of the best algorithms since it provides more accurate edge detection values for applying searching algorithm in the this work (Biswas and Sil, 2012; Catal Reis et al, 2016). In this work, canny edge detection algorithm for extracting the edge for the medical images have been used. We are also planning a further similar study on different bones' tumor.

\section{REFERENCES}

Bakotic, B. and Huvos, A.G., 2001. Tumors of the Bones of the Feet: The Clinicopathologic Features of 150 Cases, Journal of Foot \& Ankle Surgery, 40(5), pp. $277-286$.

Biswas, R. and Sil, J., 2012. An Improved Canny Edge Detection Algorithm Based on Type-2 Fuzzy Sets, Procedia Technology, 4, pp. 820 - 824 .

Canny, J.F., 1983. Finding Edges and Lines in Images, Technical Report AI-TR-720, MIT, Artificial Intelligence Laboratory, Cambridge, MA.

Canny, J.F., 1986. A Computational Approach to Edge Detection, IEEE Transactions on Pattern Analysis and Machine Intelligence, 8(6), pp. 679-698.

Catal Reis, H., Bayram, B. and Seker, D.Z., 2016. Bone Tumor Segmentation by Gradient Operators from CT Images, Selcuk International Scientific Conference on Applied Sciences (The Selçuk ISCAS 2016), 27-30 September, Antalya, Turkey. 
Doi, K., 2007. Computer-Aided Diagnosis in Medical Imaging: Historical Review, Current Status and Future Potential, Comput Med Imaging Graph., 31(4-5), pp. 198-211.

Foo, L.F. and Raby, N., 2005. Tumors and Tumor-like Lesions in the Foot and Ankle, Clinical Radiology, 60, pp. 308-332.

Giger, M.L., Huo, Z., Kupinski, M.A., Vyborny, C.J., 2000. Computer-Aided Diagnosis in Mammography. In: Fitzpatrick JM, Sonka M, editors. The Handbook of Medical Imaging, volume 2 Medical Imaging Processing and Analysis, SPIE, pp. 915-1004.

Gonzalez, R.C. and Woods, R.E., 2007. Digital Image Processing", 2nd ed., Beijing: Publishing House of Electronics Industry.

Hasbek Z., Salk I.., Yucel B., Akgul Babacan, N., 2013. Which Imaging Method to Choose for Detection of Bone Metastases? Bone Scintigraphy, CT, 18F-FDG PET/CT or MR? Bozok Med J, 3(3), pp. 44-50.

Jepson, A.D. and Fleet, D.J., Edge Detection, 2009, http://www.cs.toronto.edu/ jepson/csc2503/edgeDetecti on.pdf, 1 Nov 2015.

Kabade, A.L. and Sangam, V.G., 2016. Canny Edge Detection Algorithm, International Journal of Advanced Research in Electronics and Communication Engineering (IJARECE), 5(5), pp. 1292-1296.

Kizilkaya, A., 2008. Department of Electrical Engineering, Lecture Note, Pamukkale University, Denizli- Turkey.

Lodwick, G.S., Haun, C.L., Smith, W.E., Keller, R.F. and Robertson, E.D., 1963. Computer Diagnosis of Primary Bone Tumors. A prelim. Report, 80, pp. 273-5.

Maini, R. and Aggarwa, H., 2009. Study and Comparison of Various Image Edge Detection
Techniques, International Journal of Image Processing (IJIP), 3(1), pp. 1-11.

Mascard, E., Gaspar, N., Brugières, L., Glorion, C., Pannier, S. and Gomez-Brouchet, A., 2017. Malignant Tumours of the Foot and Ankle, EFORT Open Rev., 2(5), pp. 261-271.

Obuchowski, N.A., 2005. ROC Analysis, Fundamentals of Clinical Research for Radiologists, American College of Radiology (ACR), 184, pp. 364-372.

Ozer, D., Er, T., Aycan O.E., Oke, R., Coskun, M. and Kabukcuoglu, Y.S., 2014. May Bone Cement Be Used To Treat Benign Aggressive Bone Tumors of the Feet with Confidence? The Foot, 24, pp. 1-5.

Rice, B.M., Todd, N.W., Jensen, R., Rush, S.M. and William Rogers, W., 2014. Metastatic Calcaneal Lesion Associated with Uterine Carcinosarcoma, The Journal of Foot \& Ankle Surgery, 53, pp. 364-368.

Rong, W., Li, Z., Zhang, W. and Sun, L., 2014. An Improved Canny Edge Detection Algorithm, Mechatronics and Automation (ICMA), IEEE International Conference, 3-6 Aug. 2014, Tianjin, China.

Toriwaki, J., Suenaga, Y., Negoro, T, et al., 1973. Pattern Recognition of Chest X-Ray Images, Computer Graphics and Image Processing, 2, pp. 252-271.

Yogamangalam, R. and Karthikeyan, B., 2013. Segmentation Techniques Comparison in Image Processing, International Journal of Engineering and Technology (IJET), 5(1) (Feb-Mar)

Copyright ( $\odot$ International Journal of Engineering and Geosciences (IJEG). All rights reserved, including the making of copies unless permission is obtained from the copyright proprietors. 